\title{
Cracking Crabs Leading to Vibrio Necrotizing Fasciitis in a Male with Undiagnosed Adrenal Insufficiency
}

\author{
Ahmad Al-Abdouh, $M D^{1^{*}}$, Eileen McDonnell, $\mathrm{MD}^{2}$, Divya Mamootil ${ }^{3}$, Anas Bizanti, MD ${ }^{1}$ and Ammer \\ Bekele, $M D^{1}$
}

${ }^{1}$ Department of Medicine, Saint Agnes Hospital, Baltimore, USA

${ }^{2}$ Department of Surgery, Saint Agnes Hospital, Baltimore, USA

${ }^{3}$ Ross University School of Medicine, Bridgetown, Barbados

*Corresponding author: Ahmad Al-Abdouh, Department of Medicine, Saint Agnes Hospital, Baltimore, MD 21229, USA, Tel: +16672346000

\begin{abstract}
Necrotizing fasciitis caused by Vibrio vulnificus is a fatal systemic infection affecting mainly immunocompromised patients. A high index of suspicion is required to start the treatment immediately, including resuscitation, systemic antibiotics, and surgical debridement. We present a case of a 72-year-old male who presented after a syncopal episode due to hypotension. He was diagnosed with Vibrio vulnificus bacteremia and necrotizing fasciitis of the right arm. During his hospitalization, he was eventually diagnosed with secondary adrenal insufficiency due to persistent normal anion gap metabolic acidosis. A timely diagnosis and prompt treatment are necessary for patients with necrotizing fasciitis survival.
\end{abstract}

\section{Keywords}

Necrotizing fasciitis, Vibrio vulnificus, Septic shock

\section{Introduction}

Vibrio vulnificus is a gram-negative bacterium predominant in marine environments. It is known to cause wound infections, septicemia, and diarrhea [1]. It is the main cause of shellfish-associated deaths in the US, accounting for $8 \%$ of aerobic bacteria in the Chesapeake Bay [2]. It mainly affects patients with chronic diseases like diabetes mellitus, chronic renal insufficiency, and adrenal insufficiency due to its dependent nature on iron and transferrin reserves for exponential growth [3]. Iron is an essential element required by Vibrio vulnificus as it plays a vital role as a co-factor for several metabolic pathways. Iron acquisition and transport into the bacteria are promoted by low molecular weight compounds called siderophores that are synthesized and excreted by the bacteria [4].

Necrotizing fasciitis (NF) caused by Vibrio vulnificus is a soft-tissue infection with a high fatality rate. It is usually induced by direct invasion or contact of the wounds with contaminated seawater or raw seafood [5]. Early diagnosis and treatment of the infection are essential for patient survival [6].

Adrenal insufficiency, a predisposing factor for Vibrio species infections [3] has nonspecific symptoms and signs like loss of appetite, nausea, weight loss, weakness, and orthostatic hypotension. A delay in diagnosing it can happen and can lead to adrenal crisis, which could be lethal [7]. Infections are the main precipitating factor for adrenal crisis and other causes like physical stress such as a surgical procedure or trauma, and psychological stress [8]. We present a case of a 72-year-old Vietnamese male who presented after a syncopal episode due to hypotension. He was diagnosed with Vibrio vulnificus bacteremia and necrotizing fasciitis of the right arm and was diagnosed during his hospitalization to have adrenal insufficiency.

\section{Case Presentation}

A 72-year-old male with a past medical history significant for hypertension, asthma, and peripheral ar-

Citation: Al-Abdouh A, McDonnell E, Mamootil D, Bizanti A, Bekele A (2021) Cracking Crabs Leading to Vibrio Necrotizing Fasciitis in a Male with Undiagnosed Adrenal Insufficiency. Clin Med Rev Case Rep 8:346. doi.org/10.23937/2378-3656/1410346

Accepted: May 29, 2021: Published: May 31, 2021

Copyright: (c) 2021 Al-Abdouh A, et al. This is an open-access article distributed under the terms of the Creative Commons Attribution License, which permits unrestricted use, distribution, and reproduction in any medium, provided the original author and source are credited. 
terial disease presented to the emergency department via ambulance following a syncopal episode at home preceded by vomiting and diarrhea for the last 2 days. Upon gathering more history from the patient's son, the patient had an open wound on his right hand from scratching it on a tree a week prior, and he continued to scratch at the wound enlarging it. His right arm became swollen and red 2 days earlier to his presentation. He also had handled live blue crabs from the local Maryland Coastal Bay the evening before. His home medications include clopidogrel $75 \mathrm{mg}$ once daily, enalapril $20 \mathrm{mg}$ once daily, hydrochlorothiazide $25 \mathrm{mg}$ once daily, metoprolol tartrate $50 \mathrm{mg}$ twice daily, amlodipine $10 \mathrm{mg}$ daily, simvastatin $40 \mathrm{mg}$ daily, icosapent 1 gram once daily, tamsulosin $0.4 \mathrm{mg}$ once daily, and levalbuterol nebulizer every 6 hours as needed for shortness of breath.

Upon arrival to the emergency room, he was afebrile with a blood pressure of $98 / 54 \mathrm{mmHg}$ and later dropped to $84 / 54 \mathrm{mmHg}$ despite aggressive fluid resuscitation. He was awake, fully oriented, his right arm was erythematous from the right elbow to hand, there was a large bulla in proximal forearm extremely tender to palpation, with decreased sensation in the arm and forearm, without any discharge or crepitus (Figure 1). The remaining of the physical examination was unremarkable.

Laboratory findings on admission revealed: hemoglobin, $11.6 \mathrm{~g} / \mathrm{dL}$ (normal range $13-17 \mathrm{~g} / \mathrm{dL}$ ); white blood cells (WBC) $14.5 \mathrm{~K} / \mathrm{uL}$ (normal range 4-11 K/uL);

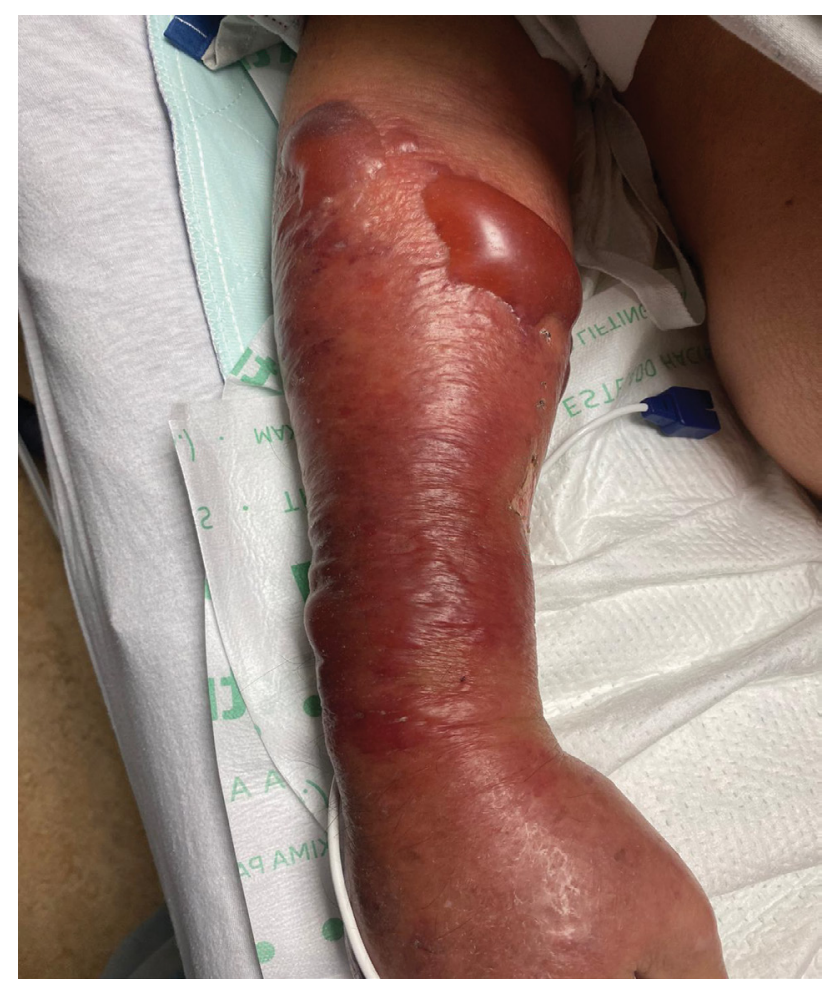

Figure 1: Right forearm upon admission. The forearm was erythematous from the right elbow to hand, with a large bulla and was extremely tender to palpation. platelets, $178 \mathrm{~K} / \mathrm{uL}$ (normal range $150-400 \mathrm{~K} / \mathrm{uL}$ ); serum creatinine, $2.1 \mathrm{mg} / \mathrm{dL}$ (baseline $0.8 \mathrm{mg} / \mathrm{dL}$ ); lactic acid $3.6 \mathrm{mmol} / \mathrm{L}$ (normal range $0.5-1.6 \mathrm{mmol} / \mathrm{L}$ ); erythrocyte sedimentation rate (ESR), $108 \mathrm{~mm} /$ hour (normal range 0-10 mm/hour); C-reactive protein (CRP), $215 \mathrm{mg} / \mathrm{L}$ (normal range $0-5 \mathrm{mg} / \mathrm{L}$ ). X-ray of the right forearm showed soft tissue densities of uncertain etiology at the dorsal and ventral aspects of the proximal forearm without any evidence of fracture or foreign bodies (Figure 2).

The next morning, his right arm had a dusky appearance with rapidly developing hemorrhagic bullae and crepitus. There was also numbness and paresthesia in the right hand. He was diagnosed with necrotizing fasciitis and taken to the operative room emergently for radical debridement. He was started on empiric broad-spectrum antibiotics coverage, including vancomycin 1.25 gm every 36 hours (for the first 3 days), piperacillin-tazobactam 3.375 gm every 8 hours (for the first 3 days), and clindamycin 600 mg every 8 hours (for the first 3 day). He was also given hydrocortisone $50 \mathrm{mg}$ every 6 hours (for 5 days, starting from the next day after admission) as he was in refractory septic shock requiring intravenous vasopressors and intravenous immunoglobulins 65 gram once daily (for 2 days, starting from the next day after admission) due to concerns of toxic shock syndrome (Figure 3). Blood and wound cultures revealed anaerobic gram-negative bacilli, Vibrio vulnificus. His antibiotics were switched on Day 3 to cefepime hydrochloride 1 gram every 6 hours and doxycycline hyclate 100 mg for a 10-day course to treat for Vibrio vulnificus bacteremia and necrotizing fasciitis. Over the next few days, he had 3 serial debridement and washouts (Figure 4). His right hand's dorsum aspect was the site of most necrosis with nonviable epidermis, dermis, and subcutaneous tis-

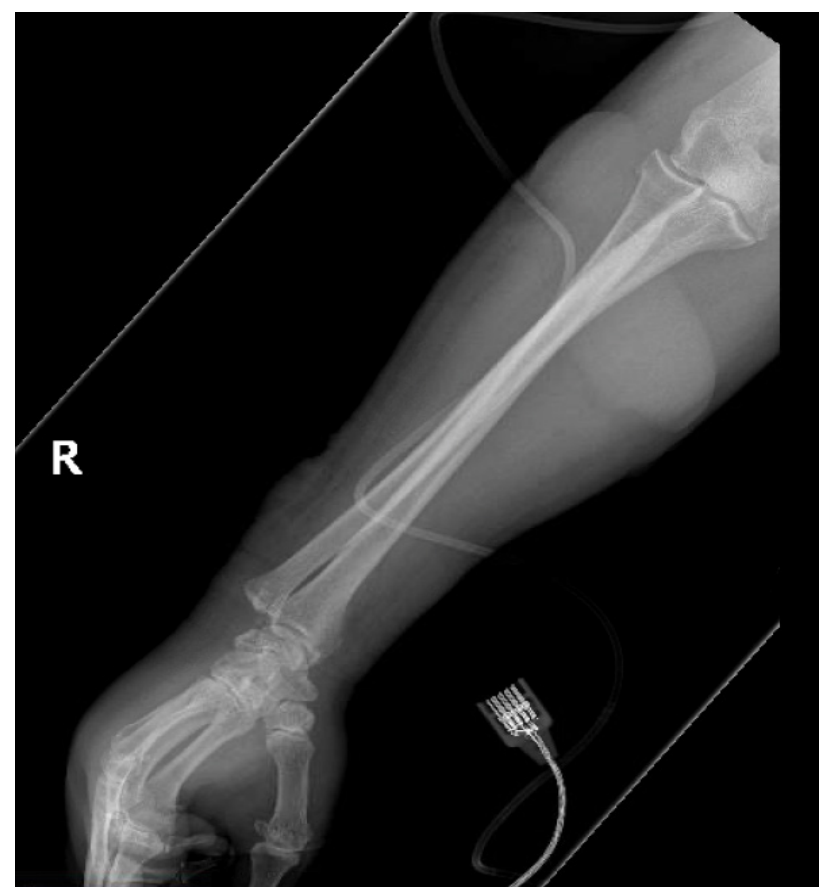

Figure 2: X-ray of the right forearm. It shows soft tissue densities at the proximal forearm. 


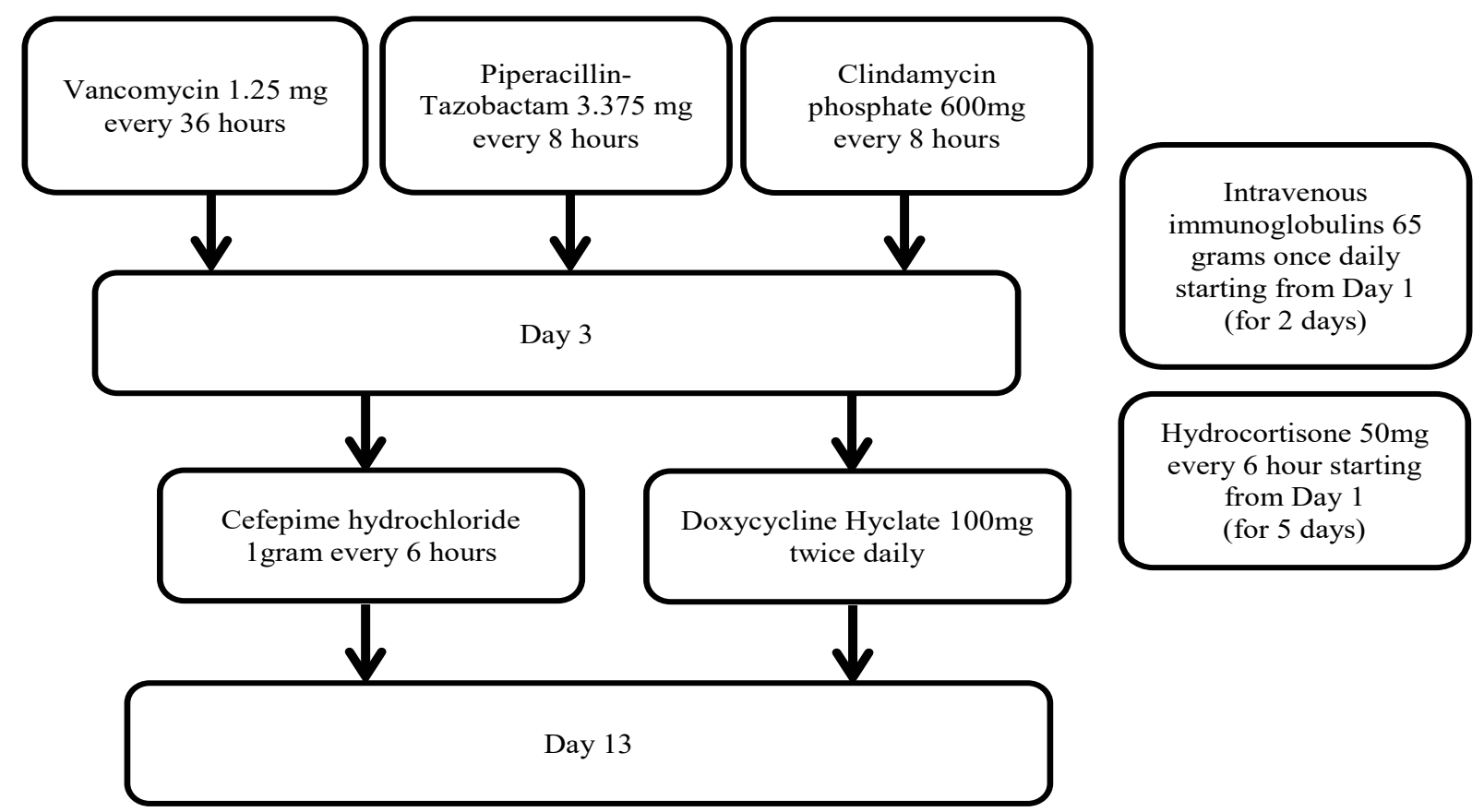

Figure 3: Schematic diagram showing the treatment plan. It shows how the patient was treated with antibiotics, hydrocortisone, and intravenous immunoglobulins during his stay in the hospital.

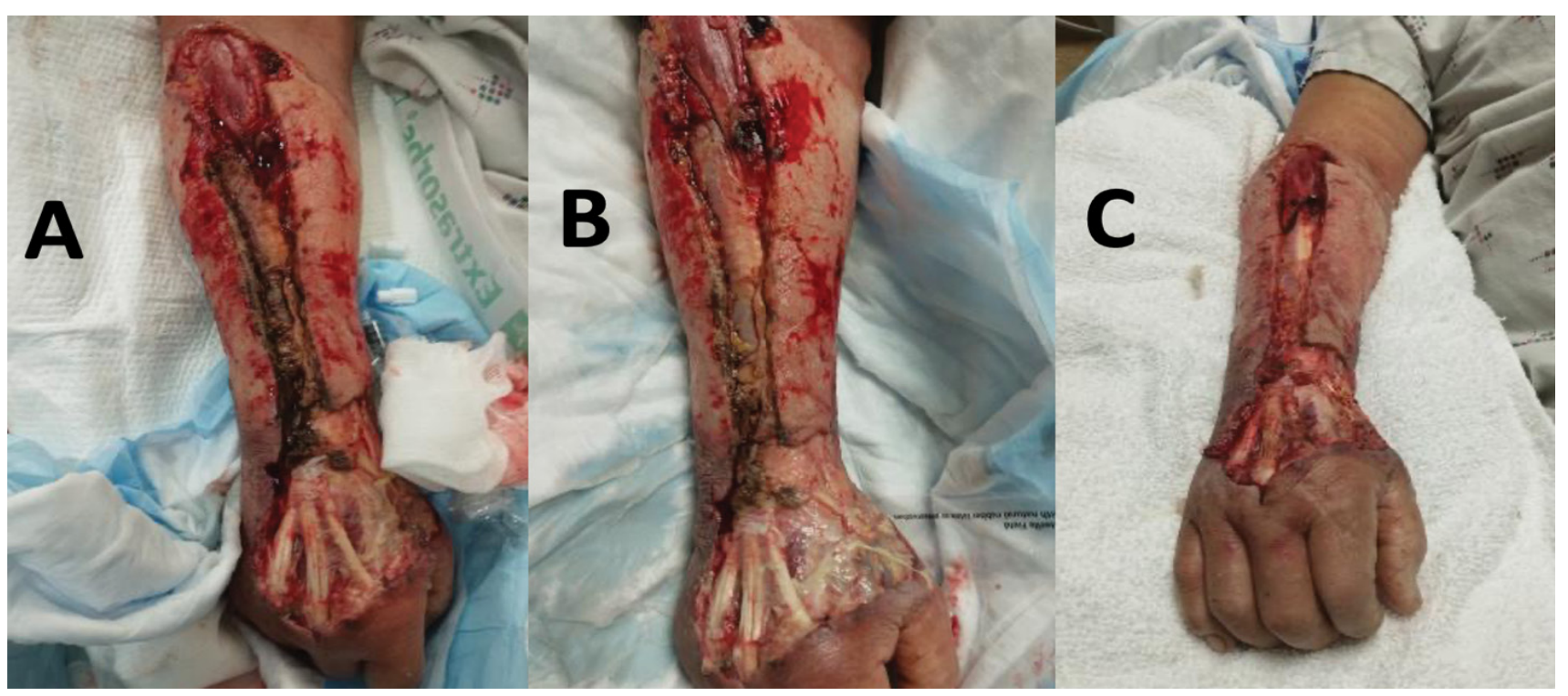

Figure 4: Debridement of the right forearm. Figure A shows the forearm after the first debridement on July $12^{\text {th }}$. Figure B shows the forearm after the last debridement on July $14^{\text {th }}$. Figure $C$ shows the forearm after application of wound vac for 48 hours on July $16^{\text {th }}$.

sues. He had a split-thickness graft placed over the right forearm, taken from the left thigh (Figure 5). A wound vac was also applied to the right arm.

He later developed worsening anemia and thrombocytopenia. His further laboratory workup revealed lactate dehydrogenase (LDH) 715 units/L (normal range $135-225$ units/L) and haptoglobins $<10 \mathrm{mg} / \mathrm{dL}$ (normal range $30-200 \mathrm{mg} / \mathrm{dL}$ ), which were suggestive of hemoIytic anemia, a complication of the patient's systemic infection. Hemolytic anemia improved gradually with antibiotics and debridement (Figure 6).

His hospital course was complicated by normal anion gap metabolic acidosis with concerns of possible adrenal insufficiency as the early morning serum cortisol level was $5 \mathrm{ug} / \mathrm{dL}$ (normal range 6.2-19.4 ug/dL). ACTH level was $20.1 \mathrm{pg} / \mathrm{mL}$ (normal range 7.2-63.3 pg/mL). A Cosyntropin stimulation test was performed (after 5 days of stopping stress dose hydrocortisone) and confirmed adrenal insufficiency. MRI of the head with contrast revealed a right-sided hyperintense mass in the pituitary gland measuring $7 \times 9 \times 7 \mathrm{~mm}$, consistent with a pituitary microadenoma (Figure 7). Upon completing the antibiotic course, the wound vac was removed, and the patient was discharged home on hydrocortisone. These 
multiple subsequent findings in acute Vibrio wound infection settings indicate the possible sequela related to such infections.

\section{Discussion}

Necrotizing fasciitis caused by Vibrio vulnificus has significant associations with seawater and marine or-

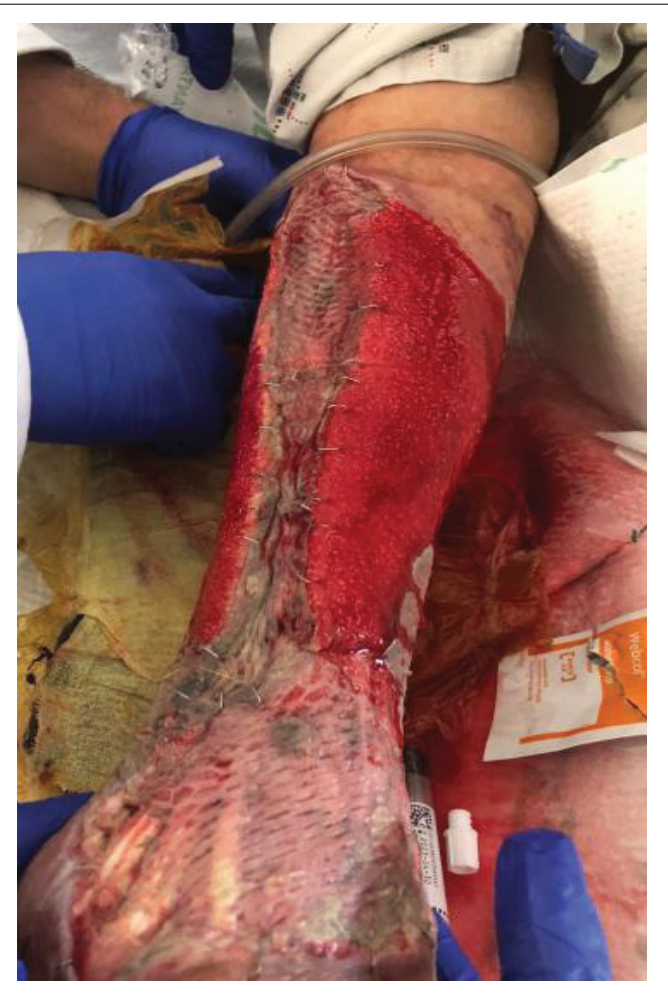

Figure 5: Right forearm after the skin graft. It shows the skin graft that was taken from the right thigh on July $20^{\text {th }}$. ganisms' contact, which was found in our case. The incidence of Vibrio vulnificus infection in the united states is estimated to be $0.01-0.03$ per 100,000 per year in 1996, and this increased to $0.04-0.05$ per 100,000 per year in 2010 [9]. With this increase in Vibrio vulnificus infections, educating people on how to handle and store seafood can decrease the incidence of these infections [9].

Clinicians should consider necrotizing fasciitis in any patient with septic shock features in addition to bullous skin lesions. The diagnosis can be confirmed by culture; the organism can be isolated from the blood or the wound. The infection usually causes necrosis of the fascial planes due to proteolytic necrosis caused by streptokinase, hyaluronidase, and cytolysin/hemolysin [10]. The infection usually involves the fascia and superficial layers while sparing deep structures like bones, muscles, ligaments, and tendons [10]. All Vibrio vulnificus strains can cause hemolysis of human erythrocytes, and this association was found as well in our patient [11].

The Laboratory Risk Indicator for Necrotising Fasciitis (LRINEC) is a tool used to assess the risk based on serum laboratory parameters. It is based on hemoglobin, white cell count, serum sodium, creatinine, C-reactive protein (CRP), and glucose. Our patient had a score of six, and scoring six or more indicates a higher risk of necrotizing fasciitis, while scoring less than 6 indicates a lower risk of necrotizing fasciitis (may treat with antibiotics and serial monitoring) [12].

The treatment of necrotizing fasciitis should be started immediately with resuscitation, antibiotics, and sur-

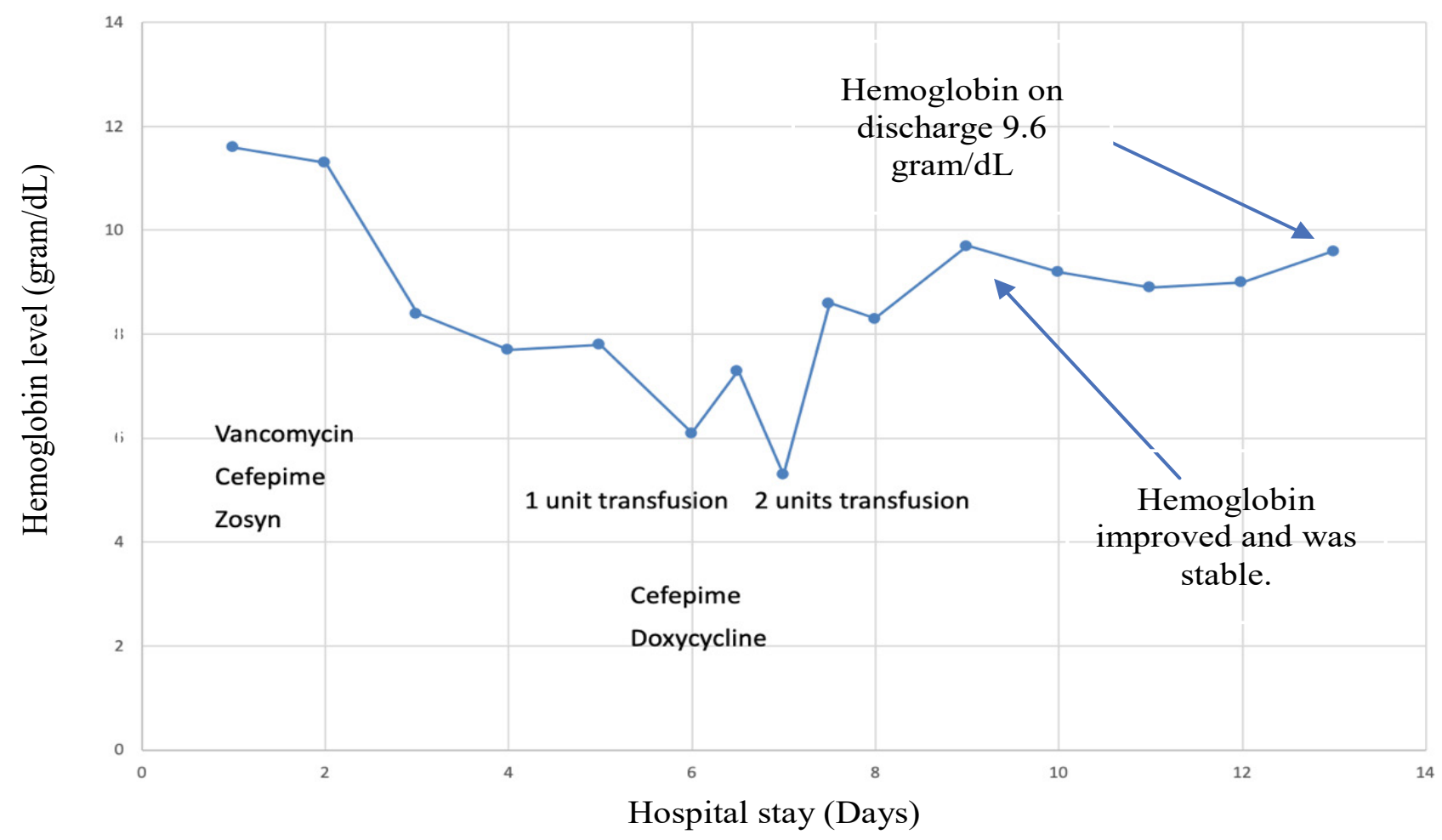

Figure 6: Hemoglobin level during hospital stay (days). The figure shows how the hemoglobin levels started to improve after starting antibiotics therapy with cefepime and doxycycline for Vibrio vulnificus. 


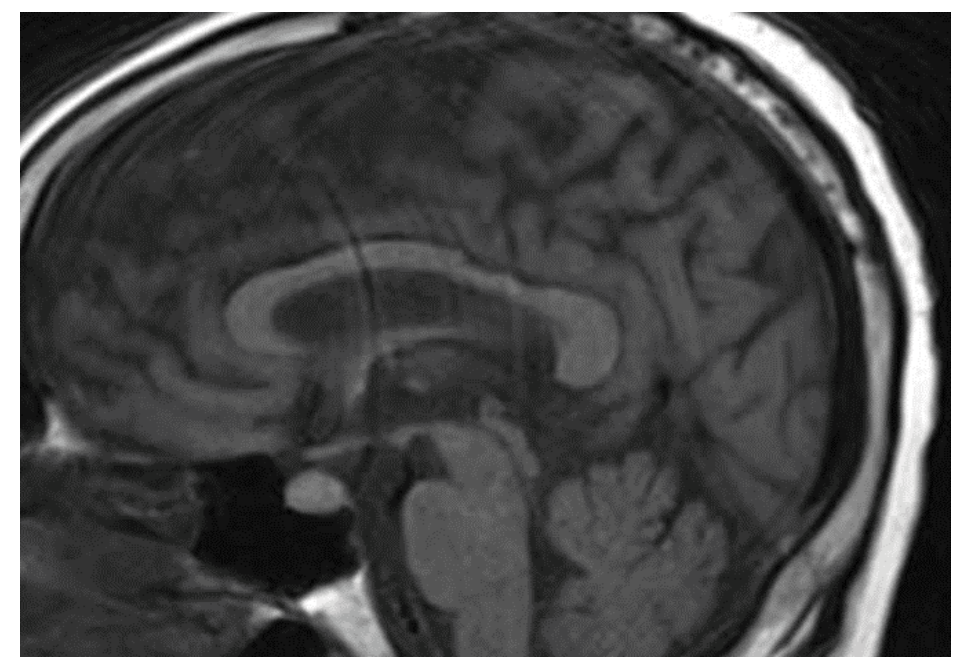

Figure 7: Brain MRI with intravenous contrast. It shows hyperintense lesion in the right aspect of the pituitary gland (pituitary microadenoma) with possible hemorrhage or complex cystic contents.

gical debridement. The preferred antibiotics regimen included minocycline or doxycycline with ceftriaxone, cefotaxime, or cefepime. Alternatively, cefotaxime and ciprofloxacin or new fluoroquinolone monotherapy can be used [13-16]. Timely extensive debridement with necrosectomy and fasciotomy should be performed as ischemia and hypoxia may compromise antibiotics delivery at the infected site. Repeated surgical debridement is necessary over the next 24 hours or later based on the infection course [17]. We used intravenous immunoglobulin treatment empirically due to suspicion of toxic shock syndrome on admission as it decreases the superantigenicity of the beta-haemolytic streptococci [18].

Vibrio vulnificus infections occur mostly in patients with immunocompromising diseases such as diabetes mellitus, liver disease, chronic kidney disease, and adrenal insufficiency. Our patient didn't have any of these risk factors on the presentation. However, having persistent normal anion gap metabolic acidosis lead to further workup that revealed secondary adrenal insufficiency due to pituitary adenoma. What distinguishes this case is the classical presentation of Vibrio vulnificus necrotizing fasciitis in settings of exposure to raw seafood and the consequences that lead to adrenal insufficiency diagnosis.

\section{Conclusion}

In conclusion, Vibrio vulnificus is a fatal systemic infection that occurs more in immunocompromised patients. A timely diagnosis is necessary to start the treatment immediately, including resuscitation, surgical debridement, and systemic antibiotics.

\section{Funding}

We did not receive any specific grant from funding agencies in the public, commercial, or not-for-profit sectors.

\section{Disclosures}

The authors don't have any relevant disclosures.

\section{Conflict of Interest}

All authors declare they have no conflict of interest.

\section{References}

1. Division of Foodborne, Waterborne, and Environmental Disease (2019) Vibrio vulnificus \& wounds.

2. Wright AC, Hill RT, Johnson JA, Roghman MC, Colwell RR, et al. (1996) Distribution of vibrio vulnificus in the chesapeake bay. Appl Environ Microbiol 62: 717-724.

3. Kuo Y-L, Shieh S-J, Chiu H-Y, Lee J-W (2007) Necrotizing fasciitis caused by vibrio vulnificus: Epidemiology, clinical findings, treatment and prevention. Eur J Clin Microbiol Infect Dis 26: 785-792.

4. Wen Y, Kim IH, Son J-S, Lee B-H, Kim K-S (2012) Iron and quorum sensing coordinately regulate the expression of vulnibactin biosynthesis in Vibrio vulnificus. J Biol Chem 287: 26727-26739.

5. Tsai Y-H, Hsu RW-W, Huang K-C, Huang T-J (2010) Laboratory indicators for early detection and surgical treatment of vibrio necrotizing fasciitis. Clin Orthop Relat Res 468: 2230-2237.

6. Jabbour G, El-Menyar A, Peralta R, Shaikh N, Abdelrahman $\mathrm{H}$, et al. (2016) Pattern and predictors of mortality in necrotizing fasciitis patients in a single tertiary hospital. World J Emerg Surg 11: 40.

7. Papierska L, Rabijewski M (2013) Delay in diagnosis of adrenal insufficiency is a frequent cause of adrenal crisis. Int J Endocrinol 2013: 482370.

8. Dineen R, Thompson CJ, Sherlock M (2019) Adrenal crisis: Prevention and management in adult patients. Ther Adv Endocrinol Metab 10

9. Newton A, Kendall M, Vugia DJ, Henao OL, Mahon BE (2012) Increasing rates of vibriosis in the United States, 1996-2010: Review of surveillance data from 2 systems. Clin Infect Dis 54: S391-S395.

10. Lazow SK (2000) Necrotizing fasciitis and mediastinitis. Atlas Oral Maxillofac Surg Clin North Am 8: 101-119. 
11. Johnson DE, Calia FM (1981) Hemolytic reaction of clinical and environmental strains of vibrio vulnificus. J Clin Microbiol 14: 457-459.

12. Wong C-H, Khin L-W, Heng K-S, Tan K-C, Low C-O (2004) The LRINEC (Laboratory Risk Indicator for Necrotizing Fasciitis) score: A tool for distinguishing necrotizing fasciitis from other soft tissue infections. Crit Care Med 32: 15351541.

13. Jang H-C, Choi S-M, Kim HK, Kim S-E, Kang S-J, et al. (2014) In vivo efficacy of the combination of ciprofloxacin and cefotaxime against vibrio vulnificus sepsis. PLoS One 9: e101118.

14. Tang H-J, Chang M-C, Ko W-C, Huang K-Y, Lee C-L, et al. (2002) In vitro and in vivo activities of newer fluoroquinolones against Vibrio vulnificus. Antimicrob Agents Chemother 46: 3580-3584.
15. Chen S-C, Lee Y-T, Tsai S-J, Chan K-S, Chao W-N, et al. (2012) Antibiotic therapy for necrotizing fasciitis caused by Vibrio vulnificus: retrospective analysis of an 8-year period. J Antimicrob Chemother 67: 488-493.

16. Trinh SA, Gavin HE, Satchell KJF (2017) Efficacy of Ceftriaxone, Cefepime, Doxycycline, Ciprofloxacin, and Combination Therapy for Vibrio vulnificus Foodborne Septicemia. Antimicrob Agents Chemother 61: e01106-17.

17. Misiakos EP, Bagias G, Patapis P, Sotiropoulos D, Kanavidis $P$, et al. (2014) Current concepts in the management of necrotizing fasciitis. Front Surg 1: 36.

18. Seal DV (2001) Necrotizing fasciitis. Curr Opin Infect Dis 14: $127-132$ 